\title{
La Première Guerre mondiale dans l'opérette de langue allemande à l'exemple de l'opérette Im Konzentrationslager (1917) de Helene Fürnkranz
}

\author{
Hélène Leclerc \\ Université Toulouse - Jean Jaurès, Centre de Recherches et d'Études Germaniques (CREG)
}

Abstract

Der Beitrag befasst sich mit der Operette einer im Lager Garaison (Hautes-Pyrénées) internierten Österreicherin. Im Ersten Weltkrieg wurden Familien deutscher und österreichischer Zivilisten in diesem französischen Lager versammelt. Helene Fürnkranz' Operette, deren Titel Im Konzentrationslager auf die offizielle Bezeichnung des Lagers zurückgreift, schildert das Leben der Internierten, wobei sie sich weitgehend auf die Biografie und die Erlebnisse der Autorin im Lager stützt. Das Werk ist insofern einzigartig, als es den Standpunkt einer Person österreichischer Herkunft auf französischem Boden während des Ersten Weltkriegs, und noch dazu jenen einer Frau, vermittelt. Darüber hinaus zählt es zu den seltenen Operetten, die den Krieg zum eigentlichen Thema und nicht nur zum Hintergrund des Geschehens machen. Angestrebt wird hier ein Vergleich dieses Opernlibrettos mit einigen Werken des deutschsprachigen Operettenkanons (Gold gab ich für Eisen und Die Csárdasfürstin von Emmerich Kálmán, Die Rose von Stambul von Leo Fall und Viktoria und ihr Husar von Paul Abraham), wobei zuerst die realistische Darstellung des Krieges untersucht wird, dann die humoristische Dimension und die Inszenierung des Konfliktes, sowie schließlich die Bedeutung der weiblichen Perspektive, die sich im Stück als vorherrschend erweist.

L'article étudie le cas d'une opérette écrite par une femme d'origine autrichienne internée au camp de Garaison (Hautes-Pyrénées) où furent regroupées des familles de civils allemands et autrichiens durant la Première Guerre mondiale. Cette opérette d'Helene Fürnkranz, dont le titre reprend la dénomination officielle du camp, évoque précisément la vie des internés en s'inspirant largement de la biographie de l'auteure et de son expérience à Garaison. L'œuvre est originale en tant qu'elle offre le point de vue d'une Autrichienne en France pendant la Première Guerre mondiale, d'une femme également, et qu'il semble s'agir d'une des rares opérettes qui fasse du conflit son sujet même et non un simple arrière-plan. En mettant ce livret en perspective avec quelques opérettes plus canoniques du répertoire de langue allemande (Gold gab ich für Eisen et Die Csárdasfürstin de Emmerich Kálmán, Die Rose von Stambul de Leo Fall et Viktoria und ihr Husar de Paul Abraham), l'article examine tout d'abord la représentation réaliste de la guerre qui se dégage de l'opérette de Fürnkranz, puis le traitement léger et humoristique qui en est fait et la mise en scène de la guerre, avant de s'interroger sur la portée de la perspective féminine, qui est centrale dans la pièce.

The article studies the case of an operetta written by a woman of Austrian origin interned at the Garaison camp (Hautes-Pyrénées) where German and Austrian civilian families were gathered during the First World War. This operetta by Helene Fürnkranz, whose title takes the official name of the camp, evokes precisely the life of the internees, drawing in large amounts on the author's biography and her experience at Garaison. The work is original in that it offers the point of view of an Austrian citizen in France during the First World War, a woman as well, and seems to be one of the few operettas that makes the conflict its very subject and not just a background. Putting this libretto into perspective with some of the more canonical operettas in the German-language repertoire (Gold gab ich für Eisen and Die Csárdasfürstin by Emmerich Kálmán, Die Rose von Stambul by Leo Fall and Viktoria und ihr Husar by Paul Abraham), the article first examines the realistic portrayal of war in Fürnkranz's operetta, then the light and humorous treatment and staging of the war, before considering the significance of the female perspective, which is central to the play.

\section{Keywords}

Erster Weltkrieg • Internierungslager • Garaison • Operette • Helene Fürnkranz (1868-1936)

Première Guerre mondiale • camp d'internement • Garaison • opérette • Helene Fürnkranz (1868-1936)

First World War • internment camp • Garaison • operetta • Helene Fürnkranz (1868-1936) 
En 2016, le Centre de Recherches et d'Études Germaniques (CREG) a lancé un programme de recherches intitulé « $\mathrm{Pa}$ trimoines nomades ", fédérant 17 chercheurs et dédié à l'histoire d'un camp d'internés civils de la Première Guerre mondiale situé à une centaine de kilomètres de Toulouse, dans le lieu de Notre-Dame de Garaison'1. À partir de septembre 1914, ce couvent sis au pied des Pyrénées abrita, en effet, un camp d'internement destiné à des familles de civils dits austro-allemands, ressortissants des pays contre lesquels la France venait d'entrer en guerre. Le camp demeura en activité jusqu'en 1919 ; il accueillit plus de 2000 internés, parmi lesquels de nombreux artistes ${ }^{2}$ et quelques célébrités, dont les plus fameuses restent le Dr. Albert Schweitzer et son épouse.

Parmi les sources et témoignages dont nous disposons aujourd'hui pour explorer cet aspect encore méconnu ${ }^{3}$ de l'histoire de la Première Guerre mondiale, figure un document singulier à plusieurs égards. II s'agit du livret d'une opérette, écrite par une femme internée au camp de Garaison, Helene Fürnkranz. Ce livret fut publié à compte d'auteur en Suisse en 1917 et vient d'être traduit en français (Inderwildi/Leclerc 2019). C'est ce document que la présente contribution voudrait examiner en le mettant en perspective avec quelques opérettes du répertoire plus canonique allemand afin d'étudier l'expression artistique de la guerre qui s'y manifeste.

Après avoir montré en quoi cette œuvre est originale, l'analyse portera sur les éléments de réalisme relatifs à la guerre qu'on peut y découvrir, puis se concentrera sur le traitement léger et humoristique de ce sujet dramatique et sur la mise en scène de la guerre, avant de se pencher sur la perspective féminine propre à cette œuvre.

\section{UNE OEUVRE SINGULIÈRE}

La première singularité de ce texte est qu'il offre le point de vue d'une Autrichienne en France, la deuxième est qu'il s'agit de celui d'une femme ${ }^{4}$. L'opérette est intitulée Im Konzentrationslager, ce qui correspond à la dénomination officielle des camps d'internement à l'époque, dont la pratique a été inaugurée par les Espagnols lors de leur guerre contre les Cubains (1896-1897) (Filhol 2004 : 9) et où le terme doit être compris au sens originel de « regroupement » (Farcy 1995 :

\footnotetext{
1 Pour une présentation détaillée de ce programme, on peut se reporter au site Internet du CREG.

2 Sur ces artistes, voir notamment les notices consacrées par Hilda Inderwildi aux photographes Henri Jacob Hensel/Henzel, Hubert Merzenich, Frédéric Thörmer, aux sculpteurs Johnny Büchs et Viktor Frisch (notice coécrite avec Hélène Florea) et au peintre et dessinateur Max Pretzfelder (Leclerc 2018 : 36-37, 69-71, 81-82, 152-153).

3 L'ouvrage pionnier consacré aux camps d'internement français est celui de Jean-Claude Farcy (1995). Sur Garaison, on peut se reporter aux travaux de Jean-Claude Vimon (1997 et 2012), José Cubéro (2017)

4 Françoise Thébaud (2013:22) a souligné l'absence des femmes dans la littérature consacrée à la Première Guerre mondiale : «Des dizaines de milliers de titres ont été écrits depuis le premier conflit mondial. La femme est absente de la plupart des thèmes abordés (buts et causes de la guerre, événements militaires et politiques, condition du soldat...) ou minorisée dans une histoire de l'arrière au quotidien interprétée par les hommes. Or la guerre affecte différemment les sexes. Guerre longue et meurtrière, la Grande Guerre est pour les hommes souffrance et traumatisme; guerre moderne, elle a besoin de la mobilisation de la nation et du soutien de l'arrière. Pour les femmes, elle ne signifie pas seulement douleur des séparations et des morts, ou maternage ; elle est aussi, par la rupture de l'ordre familial des séparations et des morts, ou maternage ; elle est aussi, par la rupture de l'
}

359). Helene Fürnkranz est une femme austro-irlandaise, née à Vienne en 1868 et morte à Mutters dans le Tyrol en 1936. Issue d'une famille de notables viennois, élevée entre l'Autriche, l'Irlande et la France, c'est une femme cultivée qui réside en région parisienne, à Bois-Colombes, depuis 1907 avec son époux, ses quatre enfants et son père. Toute la famille est arrêtée au début de la guerre et internée à Garaison à partir du 7 septembre 1914. Le père d'Helene Fürnkranz, âgé de 72 ans, sera rapatrié dès les premiers convois, le 3 novembre 1914 ; elle-même regagnera la Suisse le 9 juin 1915 avec ses filles ; son époux et son fils, mobilisables, restèrent à Garaison, le père jusqu'au 9 juin 1917, le fils vraisemblablement jusqu'à la fin du conflit. Outre cette opérette, Helene Fürnkranz fait paraître dès son rapatriement un journal consacré à son internement (Fürnkranz 1915) ${ }^{5}$.

L'opérette s'inspire très largement de la biographie de l'auteure et de son expérience à Garaison. Le premier acte nous plonge ainsi dans le quotidien d'une famille bourgeoise autrichienne installée dans les alentours de Paris ; la protagoniste est l'industrieuse jeune fille de la maison, Heidi, dont les qualités rappellent la débrouillardise qui fut celle de l'auteure à Garaison ${ }^{6}$. Heidi est fiancée à Victor. Elle vit avec son frère Rolf, son grand-père et une domestique tyrolienne, Moidl. La guerre éclate à la fin du premier acte et la famille entière est arrêtée. Le deuxième acte s'ouvre, deux jours plus tard, dans la cour d'un couvent, qui ressemble fort à celui de Garaison ; les internés s'installent et découvrent la vie au camp. Heidi doit en particulier se garder des assauts du commandant en charge du camp, un séducteur comme le veut le cliché concernant les Français, et son fiancé nourrit des plans d'évasion. L'acte se clôt sur une altercation entre Victor et le commandant. Le troisième acte se poursuit dans le camp : le projet d'évasion se concrétise avec l'aide de Heidi et des Tsiganes, Victor s'évade, Heidi avoue sa complicité, elle est emprisonnée, mais un émissaire d'une puissance neutre intervient tel un deus ex machina pour négocier sa libération. Elle peut rejoindre la Suisse. En dépit du sujet grave, il s'agit d'une pièce éminemment comique.

Le projet initial de cette contribution était de mettre cette $œ u v r e$, certes mineure, en perspective avec d'autres opérettes de langue allemande, plus connues, qui aborderaient elles aussi la thématique de la Première Guerre mondiale. C'est là qu'est apparue une troisième singularité de cette opérette. En effet, si le genre de l'opérette a pu être instrumentalisé au service de la propagande de guerre, si comme le souligne Volker Klotz (2004 $\left.{ }^{3}: 655\right)$, nombre de compositeurs se sont adonnés à l'expression d'un patriotisme chauvin dans l'opérette pendant la Première Guerre mondiale, peu d'opérettes ont finalement fait de la Première Guerre mondiale un sujet en soi. Dans la plupart des cas, il s'agit simplement d'un arrière-plan. Cela peut étonner - ou

5 Ce journal ne constitue pas un cas unique. Deux autres journaux de femmes internées à Garaison ont été retrouvés et partiellement traduits en français. Voir Köbner/Schaarschmidt (2016). Pour une mise en perspective de ces trois journaux, voir Leclerc (2020).

6 Helene Fürnkranz $(1915: 38,58,61)$ devient cheffe de chambrée le 13 septembre 1914 et est en tant que telle chargée d'enregistrer les nouveaux arrivants, plus tard, elle est promue « déléguée générale » (Generaldelegierte) par le directeur du camp. 
peut-être justement pas - car le genre de l'opérette semble avoir joué un rôle important dans la mobilisation morale à l'arrière et le divertissement des troupes au front. II y eut de nombreuses représentations d'opérettes dans les camps de prisonniers et d'internés civils, comme en témoigne par exemple une lettre du soldat Ernst Emmerich à ses parents en date du 22 novembre 19157. Une affiche annonçant la représentation d'une opérette intitulée Die Dame von Ostende, organisée au bénéfice des prisonniers de guerre allemands en Angleterre par les prisonniers civils allemands (parmi eux sans doute le compositeur et l'auteur du livret de ladite opérette) du camp de Lofthouse Park, près de Wakefield le 3 octobre 1915 constitue un autre exemple ${ }^{8}$.

Nous n'avons aucune trace d'une représentation de l'opérette d'Helene Fürnkranz à Garaison. Vu son contenu somme toute peu flatteur pour les Français, il paraît improbable que les autorités du camp en aient autorisé la représentation ; on ne sait pas non plus si l'opérette a été écrite pendant l'internement de l'auteure ou seulement après son rapatriement.

Après la fin du conflit, le sujet de la guerre semble en outre devenu tabou dans l'opérette. Faut-il en déduire qu'il s'agit d'un sujet impropre au divertissement, lequel serait la mission première et obligée du genre de l'opérette ?

Parmi les opérettes du répertoire connu qui évoquent la Première Guerre mondiale, on peut en recenser quatre en langue allemande.

La première est Gold gab ich für Eisen ${ }^{9}$ du compositeur hongrois Emmerich Kálmán (1882-1953), d'après un livret de Léon Victor, créée le 18 octobre 1914 au Theater an der Wien et que Volker Klotz (ibid. : 447) qualifie du bel oxymore de « weltkriegerische Schmachtoperette ». Un premier trait commun entre cette opérette et celle d'Helene Fürnkranz réside dans le titre qui renvoie explicitement à la guerre et qui n'a rien à voir avec les titres habituels d'opérette. Elle fut conçue dans le cadre de la commémoration des guerres de libération de 1813 et du centenaire de la Bataille des Nations. Le titre fait référence à l'appel lancé en mars 1813 par la princesse Marianne de Prusse afin que les femmes de Prusse fassent don de leur or pour financer l'effort de guerre. En échange, elles recevaient

\footnotetext{
7 "Nun bin ich also wieder ganz wo anders, wenns auch nur wenige hundert Meter von der alten Stellung ist. Andere Vorgesetzte, andere Kameraden, andere Leute, andere Gegner, anderes Gelände, anderer Boden, andere Empfangsstellen für Material, andere Verpflegung; kurz: ich muß von vorne anfangen, wo bei meinem Vorgänger alles altgewohnt am pflegung; kurz: ich muß von vorne anfangen, wo bei meinem Vorgänger alles altgewohnt am es auch - $\mathrm{Na}$, es wird bei dem vorliegenden guten Willen aller Teile mit der Zeit schon werden. Stellungskrieg - Sorgen! - Es ist unter anderm zum verzweifeln, was sich unsre obern Stellen wieder alles an Dekretieren leisten. Wenn da nicht täglich mindestens 10 Folioseiten kommen, dann preist sich alles glücklich, die Bürokratie feiert Orgien, wie sie der Frieden kaum gezeitigt hat. Und dabei gehts in Serbien: Tat auf Tat! Sieg auf Sieg! und wir - zählen alle 4 Tage unsere Patronen nach! - „Die bewaffnete Neutralität heißt unser Krieg schon bei uns (allerdings mit „Zwischenfällen“). - Man erlustieret sich in Reichweite der franz. Geschütze im Kino, Zirkus, Kasino (mit u. ohne Tafelmusik) stets aber mit Sect. Läßt sich Bücherauswahlsendungen schicken, nimmt nach Belieben ein Wannen- oder Brause-Bad Bücherauswahlsendungen schicken, nimmt nach Belieben ein Wannen- oder Brause-Bad (Freischwimmbad leider schon zu kalt). Dann mal ein bissel Scheibenschießen. Schulreiten (der oberen „Berittenen“) u. s. f. u. s. f. - Ist das vielleicht der ,Krieg”, der uns die sittliche Erneuerung bringen soll? Kino? Sect? Operetten-Potpourries? - - 》 (Museumsstiftung Post
und Telekommunikation, Bonn, Inventarnummer 3.2011.3530).

8 Cette affiche est consultable à l'adresse suivante : https://digital.staatsbibliothek-berlin de/werkansicht/?PPN=PPN749938285 [Die Dame aus Ostende: Operette in 2 Akten, Text von Hans Wolf, Musik von Karl Westhoff ... ; Wohltaetigskeitsvorstellung zu Gunsten der Kriegsgefangenen deutschen Soldaten in England veranstaltet von den deutschen Civilgefangenen in Lofthouse Park Wakefield Nordlager den 3. Okt. 1915]. Nos recherches n'ont pas (encore) permis de retrouver ni le livret ni la partition de cette opérette. Sur le camp de Lofthouse Park, voir notamment https://ruhlebenlofthouse.com/ (consulté le 09/02/2021).

9 Sur cette opérette, voir l'analyse de Micaela Baranello (2014 : 253-277).
}

un anneau de fer. Ce souvenir est réactivé en Autriche lors de l'entrée en guerre en 1914. Cependant, si le titre ancre bien l'opérette dans le contexte de la guerre, la réalité de la guerre n'est pas vraiment décrite dans l'opérette de Kálmán, contrairement à l'opérette de Fürnkranz : l'action se déroule à l'arrière et l'accent est mis sur des intrigues familiales et le retour attendu du soldat, la mort est bannie tandis que chez Fürnkranz elle rode, ne serait-ce que par l'allusion à celle du Tsigane Janosch (II, 6).

La deuxième opérette est Die Csárdasfürstin du même compositeur. Elle fut créée à Vienne, au théâtre Johann Strauss, le 17 novembre 1915 ; le livret est signé de Leo Stein et Béla Jenbach. II s'agit d'une histoire d'amour a priori impossible entre un aristocrate viennois et une chanteuse de cabaret. L'opérette remporta un immense succès, d'abord à Vienne, pendant toute la durée de la guerre et dix ans après, puis un succès international. Toutefois, l'écriture et la composition avaient été commencées en 1913, donc avant la guerre. Concernant la genèse de l'œuvre, les avis des spécialistes divergent : certains considèrent qu'elle est antérieure au début de la guerre et qu'il serait vain de tenter d'y débusquer des allusions au conflit ; d'autres, comme Micaela Baranello (2014 : 288), affirment que seul l'acte I était prêt avant la guerre, que le second date de l'été 1915 et que tous les textes de chansons ont été écrits par Stein au milieu de la guerre. II est certain qu'on n'y trouve nulle allusion directe à la guerre mais Micaela Baranello (ibid.) souligne que cette opérette présuppose « un monde d'incertitude énorme » (a world of enormous uncertainty), et l'on peut raisonnablement considérer que les tensions internationales en 1913 aient déjà instauré une forme de "culture de guerre " ${ }^{10}$, que Karl Kraus dénonçait du reste dès $1912^{11}$.

La troisième opérette est Die Rose von Stambul de Leo Fall (1873-1925), créée le 2 décembre 1916 à Vienne, au Theater an der Wien, d'après un livret de Julius Brammer et Alfred Grünwald. L'histoire se déroule dans l'Empire ottoman. Là non plus, le thème n'est pas la Première Guerre mondiale, mais le choix du lieu de l'action pourrait avoir été influencé par le contexte de la guerre puisque la Turquie était alliée de l'Allemagne. Volker Klotz $\left(2004^{3}: 353\right)$ indique que l'action se déroule « avant la Première Guerre mondiale » et établit donc un lien implicite avec celle-ci. Il s'agit de nouveau d'une histoire d'amour entre la belle Kondja Gül, fille du pacha, « la rose d'Istambul », qui doit épouser le fils du ministre Achmed Bey mais qui est éprise du poète André Léry ; ce qu'elle ignore, c'est que les deux hommes ne sont qu'une seule et même personne. L'opérette s'inscrit dans la tradition mozartienne de la turquerie.

10 Cette notion, élaborée dans les années 1990 par les historiens français Annette Becker et Stéphane Audoin-Rouzeau notamment, est associée à la thèse d'un " consentement " patriotique à la guerre de la part de la population, qui permettrait d'expliquer comment les combattants " ont tenu » pendant quatre ans. Cette thèse a suscité de vifs débats et a été contestée par les historiens membres du CRID 14-18, qui, s'intéressant aux témoignages des combattants, considèrent que ce " consentement " à la guerre relève davantage des élites politiques et culturelles. Sur ces notions et débats, voir entre autres : Audoin-Rouzeau/ Becker A. (2000), Audoin-Rouzeau/Becker J.J. (2004).

11 Micaela Baranello (2014 : 249) cite l'article « Und in Kriegszeiten » publié par Karl Kraus dans Die Fackel 14, Nr. 363-65 (décembre 1912). 
La quatrième et dernière opérette est postérieure au conflit. Créée le 7 juillet 1930, d'après un livret original hongrois d'Emmerich Földes, remanié pour la version allemande par Alfred et Fritz Löhner-Beda, Viktoria und ihr Husar de Paul Abraham (1892-1960) présente un arrière-plan directement lié à la guerre puisque deux des personnages, dont le grand amour de jeunesse de l'héroïne qui refait surface, sont des prisonniers de guerre évadés de camps russes de Sibérie. On peut peut-être mentionner enfin une opérette en croate, Mala Floramye, du compositeur croate Ivo Tijardović (18951976), créée en 1926 et dont l'action se situe à Split et Marseille avant, pendant et après la Première Guerre mondiale. Outre le fait qu'il ne s'agit pas d'une opérette en langue allemande, la guerre n'y est, en dépit du cadre temporel explicite, que l'arrière-plan et non le thème. Quant à Rund um die Liebe d'Oscar Straus (1870-1954), créée le 9 novembre 1914 au Johann Strauss-Theater de Vienne, soit à peu près au même moment que Gold gab ich für Eisen, il s'agit d'une opérette à contre-courant de celle-ci : l'action se déroule avant 1914, le bonheur et la légèreté y sont exaltés et l'œuvre s'inscrit, d'après Volker Klotz (ibid. : 675), « contre le pathos de la grandeur et de la durée », comme l'indiquent par exemple les titres des numéros « Ein Schwipserl möcht' ich haben » et « Es gibt Dinge, die muss man vergessen ». Cet état des lieux confirme la singularité de l'œuvre d'Helene Fürnkranz. Nous nous appuierons uniquement sur l'analyse du livret car la partition n'a pas été retrouvée ; une hypothèse probable est que celle-ci n'a jamais existé et que les parties chantées $^{12}$ étaient censées l'être sur des airs connus ${ }^{13}$. La présence de Tsiganes dans la pièce laisse volontiers imaginer une coloration musicale tsigane (Inderwildi/Leclerc 2019 : 11-12), telle qu'on peut la trouver dans les opérettes de Lehár ou Kálmán. Sans doute Fürnkranz a-t-elle songé à l'une ou l'autre de ces opérettes, comme nous le montrerons plus loin.

\section{UNE REPRÉSENTATION RÉALISTE DE LA GUERRE}

Le titre de l'opérette, Im Konzentrationslager, est d'emblée révélateur de l'intention de témoigner d'une réalité vécue. Helene Fürnkranz ne sacrifie pas cette intention à la tradition de l'opérette qui ancre volontiers son action dans une région ou une époque éloignée (Klotz 2004 : 65). II s'agirait d'une nouvelle particularité de cette opérette, qui, toutes proportions gardées, s'inscrirait sur ce point dans le sillage de La Vie parisienne d'Offenbach, Volker Klotz (ibid. : 106) soulignant en effet que les opérettes évoquant directement leur époque sont minoritaires ${ }^{14}$. L'ambition première est bien de rendre compte d'une expérience vécue. Aussi le quotidien

12 Les parties chantées sont au nombre de 23 , soit un chiffre supérieur à la tradition de l'opérette viennoise : 8 airs, 7 duos, un trio, 3 morceaux d'ensemble, 3 chœurs et un finale. Les timbres de voix ne sont pas indiqués.

13 Cette hypothèse nous est suggérée par l'exemple de l'opérette de Germaine Tillion, Le Verfügbar aux Enfers, écrite au camp de Ravensbrück en 1944. Voir Inderwildi/Leclerc (2019: 12)

14 À propos de La Vie parisienne, Louis Bilodeau (2019 : 89) souligne aussi que cette opérette « fait figure d'exception », Offenbach « abord[ant] rarement des sujets dont l'intrigue est contemporaine ». au camp, par ailleurs décrit de manière circonstanciée dans le journal d'Helene Fürnkranz, est-il fidèlement représenté et l'opérette peut se concevoir comme une réécriture du journal, certaines scènes étant réemployées, mais cette fois sur un mode comique. On retrouve ainsi la scène de la distribution de paille aux internés à leur arrivée au camp, l'évocation de la discipline militaire imposée au son du clairon et rythmée de multiples corvées, l'existence d'une prison et la description, récurrente, de scènes de repas (Inderwildi/ Leclerc 2019 : 12). Participent également de ce parti pris réaliste l'évocation des échanges d'internés par l'intermédiaire d'une puissance neutre, la Suisse en l'occurrence, qui n'est toutefois pas nommée, des évasions (il y eut de nombreuses évasions depuis Garaison vers l'Espagne), de la livraison de colis aux internés, de la présence de Françaises parmi les internés, détenues parce qu'elles ont épousé un Allemand et sont de ce fait devenues allemandes, ainsi que la représentation des Tsiganes, sur laquelle il convient de s'attarder davantage parce qu'elle a plus directement trait au genre de l'opérette viennoise. Depuis Le Baron tsigane de Johann Strauß fils (1885), le motif tsigane est effectivement récurrent dans l'opérette viennoise, il fut considérablement développé ensuite avec Lehár (Zigeunerliebe, 1910) ou Kálmán (Der Zigeunerprimas, 1912, Die Csárdasfürstin, 1915, Gräfin Mariza, 1924). Chez Fürnkranz, le grand-père des protagonistes est d'ailleurs " baron », certes non tsigane, mais il y a sans doute dans ce titre de noblesse un clin d'œil à Strauß. Comme l'écrit Bernard Banoun (1998: 82), « les Tziganes sont représentés, traditionnellement et ici [dans Le Baron tsigane] en particulier, comme un peuple 'naturel' à la fois sauvage, impulsif et non corrompu par la société ». Chez Helene Fürnkranz, les Tsiganes sont qualifiés de « freies Volk» (III, 4) et incarnent l'idéal de liberté bafoué par les puissances belligérantes. Leur rôle est double : ils ont une fonction dramatique d'adjuvant (c'est la Tsigane Maritza qui fournit la tisane destinée à endormir le garde pour permettre l'évasion du protagoniste Victor) et les chants et la musique tsiganes sont, comme dans Die Csárdasfürstin de Kálmán, convoqués pour dissiper la tristesse des personnages. Comparons deux extraits de Die Csárdasfürstin et de Im Konzentrationslager:

Die Csardasfürstin (III, N 14, trio):

Nimm, Zigeuner, deine Geige,

Laß seh'n, was du kannst!

Schwarzer Teufel, spiel' und zeige,

Wie dein Bogen tanzt!

Spiel' ein Lied, das weint und lacht,

Spiele, bis der Bogen kracht,

Spiele, bis heranbricht hell das Morgenrot,

Spiele, Betyar, schlage mir die Sorgen tot!

Jaj mamám, Bruderherz, ich kauf' mir die Welt!

Jaj mamám, was liegt mir am lumpigen Geld!

Weißt du, wie lange noch der Globus sich dreht,

Ob es morgen nicht schon zu spät! 


\section{Im Konzentrationslager (III, 4)}

Maritza (zu den Zigeunern): Freunde, ein Lied, ein Tanz, um die Traurigkeit zu verscheuchen.

$$
[\ldots]
$$

Chor:

$$
\begin{aligned}
& \text { Durch Länder wir streichen } \\
& \text { In's Blaue hinein. } \\
& \text { Was man nie kann erreichen } \\
& \text { Das lockt uns allein! } \\
& \text { O herrliche Gabe } \\
& \text { Zu sein ohne Dach! } \\
& \text { Wo man einst uns begrabe, } \\
& \text { Wer frägt auch darnach. }
\end{aligned}
$$

Les deux dernières lignes de l'extrait de Kálmán, qui rappellent les deux dernières du chœur des Tsiganes chez Fürnkranz, sont relevées par Micaela Baranello (2014 : 249) qui y décèle une allusion au contexte de guerre. La première scène de l'opérette de Fürnkranz semble y faire écho, l'héroïne chante en effet :

Die Welt ist so schön - wunderschön,

Die Menschen so lieb - ach so lieb!

Und ich, ich bin glücklich - so glücklich,

Wenn's nur immer so bliebe - so blieb!

On peut bien sûr interpréter la présence des Tsiganes comme une recherche d'exotisme. Volker Klotz (2004³ : 220) parle ainsi, à propos de l'opérette Viktoria und ihr Husar, d'un « idiome de la Puzsta » qu'on pourrait retrouver chez Fürnkranz dans ce chant tsigane évoquant « le peuple libre [qui] chemine à travers prés » (Inderwildi/Leclerc 2019 : 56). Toutefois, chez cette dernière, la représentation des Tsiganes est motivée par les faits historiques : les Tsiganes ont été internés pendant la Première Guerre mondiale, Tsiganes alsaciens-lorrains, dont le cas a été étudié par l'historien Emmanuel Filhol (2004), mais aussi Tsiganes français, jugés suspects et dangereux. À Garaison, camp d'internés civils austro-allemands, il s'agit de Tsiganes alsaciens-lorrains.

L'exemple des Tsiganes montre particulièrement bien comment Fürnkranz conjugue volonté de témoigner d'une réalité historique et désir de divertir en s'appropriant les codes du genre de l'opérette.

\section{LE TRAITEMENT LÉGER ET HUMORIS- TIQUE D'UNE RÉALITÉ DRAMATIQUE : LA MISE EN SCÈNE DE LA GUERRE}

L'opérette peut apparaître comme le genre de la dédramatisation par excellence. C'est du moins ce que souligne Moritz Csáky (1998²:66) : "Denn das, was in der Realität des sozialen Lebens bitterer Ernst war, das konnte auf der Bühne ins Leichte, Heitere, Komische, ja Satirische verfremdet und aus der Perspektive des verdunkelten, anonymen
Zuschauerraumes betrachtet, verlacht, verspottet und kritisiert werden. "

L'historien autrichien considère que la mise en cause de la politique, si elle peut surgir dans les opérettes, ne se fait en général que de manière inoffensive et déguisée (ibid. : 73). Et de citer l'exemple, régulièrement mis en avant, de La Veuve joyeuse. Mais on peut aussi songer, avec Volker Klotz $\left(2004^{3}: 35\right)$, à l'opérette d'Offenbach : « So spielt die Offenbachiade, sei sie vom Namensgeber selber oder von seinen Nachfolgern, immer wieder wuchtige Schrecknisse aus, um sie listig und munter zu überspielen. Zerstörerische Kräfte der Erfahrungswelt, je schlimmer, je besser, beschwört sie herauf, um sie mit lebenslustigen Gegenkräften zu durchkreuzen. Das Publikum soll lachen. Aber nicht über harmlose Nebensachen, sondern darüber, dass gefährliche Hauptsachen sich bezwingen lassen ${ }^{15}$. "

L'opérette de Fürnkranz obéirait à ce schéma. La scène d'exposition installe d'emblée un climat de trouble : la chanson de Heidi célébrant la douceur estivale est brutalement interrompue par le baron poursuivi par une abeille, la quiétude est ensuite perturbée par l'évocation de l'accident de voiture de Rolf et de Victor. Le motif de la guerre ne tarde pas à surgir, d'abord suggéré par un vocabulaire militaire employé sur le ton de l'humour. Victor déclare à sa fiancée Heidi : «Werde dich schon abrichten Schatz » $(\mathrm{I}, 3)$, terme repris plus tard par le commandant du camp à propos de la même Heidi (« Wie schwer abzurichten die Weiber doch sind ! " II, 9). Le terme " guerre » en tant que tel apparaît à la toute fin de la scène I, 4 et tombe comme un couperet : " Ja, Kinder, Ihr lacht und singt... - alles deutet auf Krieg ", dit le baron. Toutefois, la dimension dramatique est immédiatement atténuée par l'entrée en scène du personnage comique et grotesque de la domestique tyrolienne Moidl qui s'empare du terme : « Krieg, sog'ns, Grä' Herr ! Jessas Maria ! und ich hob so a heilige Angst vorn Krieg seit i a kloans Kind bin! Je - je - je, das ist so eppes Gefährliches - » $(I, 5)$. Le thème se trouve ainsi traité sur un mode comique qui n'en interdit pas moins l'expression d'une réalité crue, celle du viol des femmes par les soldats. Au début de l'acte II, lorsqu'est évoquée l'arrivée des internés au camp au terme d'un éprouvant périple dans des wagons à bestiaux, la description brute du traitement de ces populations civiles et de leur animalisation ( "Gestossen, gerüttelt, gebeutelt, geschüttelt [...] Wie Kälber, wie Kühe, wie Ochsen, wie Schweine » II, 2) est à nouveau tempérée par l'irruption du grotesque, culminant dans l'allusion aux ronflements des internés :

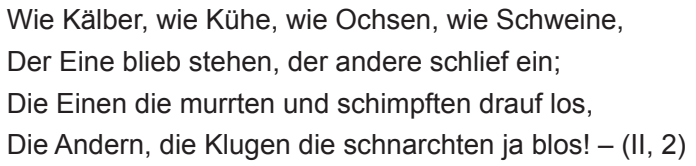

La combinaison entre l'expression d'une réalité dramatique et son traitement comique est parfaitement illustrée par

\footnotetext{
15 La réception posthume d'Offenbach a du reste souligné la dimension politique de ses opérettes. Citant Jules Vallès et Jean Richepin, Jean-Claude Yon (2000 : 613) fait du compositeur un « précurseur malgré lui de la Commune ».
} 
l'alliance de mots «Viehcoupé » (II, 2) désignant le wagon à bestiaux ayant conduit les internés au camp. De manière récurrente, le registre noble se trouve associé au registre vulgaire et ainsi dévalorisé, à l'exemple du ténor interné réduit à faire bouillir la soupe :

Vor einem begeisterten Publikum

Sang ich vor kurzem Opernlieder

Arien hold und hehr -

Jetzt, s'ist doch wahrhaftig dumm

Bin ich nur ein Suppensieder!

Soupe aux pommes de terre! (II, 5)

Les rimes de cette aria soulignent l'incongruité de son sort et sa déchéance : Publikum - dumm ; Opernlieder - Suppensieder; hehr-pommes de terre.

La construction dramatique contribue à désamorcer les scènes tristes (comme la scène II, 6 où est évoqué l'enfant orphelin et souffrant de la Tsigane Maritza) en les faisant suivre d'une scène comique (II, 7 est celle du duo de séduction entre le commandant et l'Allemande Lolotte, devenue française par son mariage). La représentation des Français est un autre élément du comique de l'opérette. Ainsi le commandant du camp est-il présenté comme une caricature de séducteur, « petit coq français » (« der französische Gockelhahn ») (III, 1), dont le courage se dégonfle instantanément à l'annonce de l'arrivée de son épouse. Est brocardée aussi la bêtise de la gendarmerie française qui procède à l'arrestation du baron à Paris dans une scène de quiproquo propre au genre, puisque les gendarmes, en possession d'une lettre écrite par celui-ci, l'accusent de vouloir vendre "le peuple français » aux Allemands $(I, 6)$; ce " peuple » (« Volk ») est en réalité une colonie d'abeilles. Contrairement à son journal d'internement où Helene Fürnkranz escamote toute critique directe à l'égard des Français et où la critique passe par la description, qui se veut objective et circonstanciée, des conditions et de la durée de l'internement, le support artistique et fictionnel de ce genre qui a vocation à dédramatiser offre ainsi de savoureuses ressources satiriques.

Outre les éléments ressortant du genre de la comédie, les emprunts à d'autres opérettes et l'intertexte apparaissent chez Fürnkranz comme autant de biais de la dédramatisation et de l'esthétisation du témoignage. Ainsi la veuve Lolotte renvoie-t-elle à la Veuve joyeuse de Lehár ; Lolo est par ailleurs le nom d'une des grisettes de cette opérette. Les duos de séduction, tels ceux de Rolf et Lolotte (II, 4), de Lolotte et du commandant (II, 7), ou celui de Heidi et du commandant (II, 9) peuvent en rappeler de plus fameux comme ceux entre Sylva et Edwin dans Die Csárdasfürstin (numéros 3, 9 et 12) ou entre Boni et Stasi dans cette même opérette (numéro 11), ou encore entre Fridolin et Midili dans Die Rose von Stambul (numéros 4 et 9) ou entre Kondja et Achmed (numéros 9 et 14), sans parler, toutes proportions gardées, du célébrissime duo « Lippen schweigen » de La Veuve joyeuse. La référence à la valse (Heidi se promet de dompter le commandant et de lui apprendre à danser la valse viennoise II, 5), ingrédient essentiel de l'opérette viennoise, est aussi un moyen de désamorcer l'inquiétude des internés quant à l'attitude du commandant. Enfin, on peut lire dans l'air du commandant (II, 8) une parodie du célèbre air de Boni « Ganz ohne Weiber geht die Chose nicht » dans Die Csárdasfürstin (numéro 4) :

Aus ist's mit der Liebe bei mir für allemal.

Schau kein Mädel mehr mir an,

Schau mir keine an!

Wenn auch tausend Herzen brechen, ist mir ganz egal.

Über alle Weiblichkeit

Mach' ich einen Strich,

In der schönsten Blütezeit

Zieh' zurück ich mich!

Mein Entschluß steht felsenfest:

Mit der Liebe ist es "Rest".

Doch:

Ganz ohne Weiber geht die Chose nicht!

Ganz ohne Sonne blüht die Rose nicht!

Drum hie und da, so einmal noch -

Da küß ich doch!

Da küß ich doch!

Ganz ohne Weiber geht die Chose nicht

Ganz ohne Sonne blüht die Rose nicht!

Drum will ich nichts verschwören,

Will, Mädels, euch gehören -

Schuft, wer sein Wort jetzt noch bricht! [...]

Im Konzentrationslager (II, 8):

Als ich ernannt zum Kommandant,

Von einem Internierten Lager,

Dacht' ich: mein Los ist nicht famos!

Die Unterhaltung wird wohl mager!

Doch siehe da!

Um mich herum,

Sind liebliche Gestalten,

Und über diese Wesen hold,

Muss schalten ich

Und walten. -

Doch hätte wieder ich die Wahl,

Nicht Weiber wollte ich regieren!

Leichter ist es tausendmal

Ein Männerregiment zu führen,

Die Schönen haben für Ordnung nicht Sinn

Sie lachen und schwatzen bei jedem Unsinn,

Von Pünktlichkeit ist keine Spur!

Bei Tadel lachen sie ja nur!

Und schimpf ich, so sehen sie mich an

Mit vorwurfsvollen Blicken,

Die mich bestricken!

So dass ich dann

Nicht streifen kann:

Ich bin doch ein geplagter Mann.

Du reste, n'y a-t-il pas un écho à ce titre dans l'usage que fait Lolotte de l'expression française pour qualifier l'amour (« Die Knospe ist lieb, doch die blühende Ros'n / Sie duftet berauschend, ist ganz autre chose », II,4) ? 


\section{4 « GANZ OHNE WEIBER GEHT DIE CHOSE NICHT » : LA PERSPECTIVE FÉMININE}

Les femmes, et la condition féminine, sont au cœur de toutes ces opérettes évoquant la guerre à des degrés divers. Leur titre met souvent en valeur une figure féminine (Die Csárdasfürstin, Die Rose von Stambul, Viktoria und ihr Husar) ; le thème de l'émancipation féminine est même porté par Die Rose von Stambul. Volker Klotz $\left(2004^{3}: 65\right)$ a résumé ce rôle des femmes dans l'opérette de la formule « Frauen am Ruder, zeitweilig auf Gegenkurs » et l'œuvre de Fürnkranz n'échappe pas à ce constat, on peut même la lire comme un manifeste féministe (Inderwildi/Leclerc 2019 : 15). Ce sont les figures féminines (Heidi, Lolotte, Maritza et dans une moindre mesure Moidl) qui portent l'action et les personnages masculins apparaissent comme des pantins ridicules : le baron, âgé, dépassé par les événements ; le jeune Rolf, troublé par la découverte du sentiment amoureux ; Victor, dépendant des initiatives de sa fiancée Heidi qui organise son évasion ; même le commandant du camp est la proie de son goût excessif pour la gent féminine et de la crainte que lui inspire son épouse. Plus profondément, l'opérette développe une critique de la barbarie masculine, portée par le personnage comique de la domestique tyrolienne Moidl, avatar de la " lustige Figur " du théâtre populaire viennois auquel appartient d'énoncer, certes avec humour, certaines vérités. Moidl, évoquant sa grande crainte qui est d'être « séduite », aborde ainsi le sujet du viol.

L'œuvre pose également la question du pacifisme féminin. Si on ne peut parler d'exaltation de la guerre dans l'opérette de Fürnkranz, il y a néanmoins une légitimation du combat et un désir de victoire, interprétée comme légitime, et ce désir est porté par la protagoniste féminine Heidi :

\author{
Und nach langem, blut'gem Ringen, \\ Kommt die siegesfreud'ge Ruh, \\ Jubelnd werden wir dann singen, \\ Freiheit! Uns gehörst jetzt du! \\ Uns're Rechte kühn verteidigt, \\ Mit dem Schwerte in der Hand, \\ Stark und stolz in schwerem Kampfe, \\ Freies Volk in freiem Land. (Fürnkranz $1915: 36$ ).
}

Une forme de bellicisme point, perceptible dans ce désir de victoire, qui n'est pas seulement un désir de liberté. Le finale est du reste révélateur de la perspective allemande : quatre personnages chantent à l'unisson la liberté, mais les Français (Lolotte et le commandant) en ont une conception très légère (pour eux, il s'agit de liberté amoureuse), tandis que Heidi et Rolf songent à la liberté politique et physique de leurs compatriotes et de leur pays. L'opérette se révèle vectrice d'une militarisation des valeurs féminines telle que l'avait vue Andreas Latzko (1918: 7-9) : « So hat die Mentalität der Frau sich gewandelt. [...] Im vierundvierzigsten Kriegsmonat, hinter Millionen deutschen und Gott weiss wie vielen Menschenleiden, stehen Frauen und wollen den Sieg. Nichts als den Sieg. Den Triumph des Starken, Unerbittlichen, über den Schwachen, Wehrlosgewordenen, um jeden, selbst um den Preis ihres Glücks! »

Les femmes constituèrent d'ailleurs une cible de la propagande dans les opérettes de l'époque (Baranello 2014 : 261).

\section{CONCLUSION : L'OPÉRETTE ENTRE PROPAGANDE ET DÉNONCIATION DE LA GUERRE}

On ne saurait évidemment mettre sur le même plan l'opérette de Helene Fürnkranz et les quatre autres opérettes mentionnées plus haut : elles diffèrent à la fois en intention et en qualité. II n'en reste pas moins que cette $œ u v r e$ mineure offre aussi l'occasion de redécouvrir de plus grands textes et de les réexaminer à l'aune de leur portée politique.

Selon Moritz Csáky (1998² : 34-35), la dimension politique de l'opérette serait en effet sous-estimée ; il y voit « un miroir de la société et de la politique » (ibid. : 62) ou un miroir des mentalités (ibid. : 25). Dans l'opérette de Fürnkranz, la dimension politique est évidente ; il n'y a chez elle nul escapisme. La réalité crue de la guerre est dite, la mort est présente, la barbarie dénoncée. Mais cette opérette, qui prend explicitement la guerre pour thème, a échoué dans sa réception ; on ignore si elle a même été représentée un jour. Ce thème cicatriciel serait-il irreprésentable, même déplacé à la problématique moins rude de l'internement?

\section{Bibliographie}

\section{CEuvres de Helene Fürnkranz}

Fürnkranz, Helene (1915), In französischer Kriegsgefangenschaft. Momentaufnahmen aus dem Leben einer Austro-Boche-Familie in Paris, Flers (Normandie), Garaison (Pyrenäen), Aarau, Separatabdruck aus dem Aargauer Tagblatt.

Fürnkranz, Helene (1917), Im Konzentrationslager. Operette in 3 Akten, Aarau, Selbstverlag.

Inderwildi, Hilda / Leclerc, Hélène (2019), Helene Fürnkranz. Une opérette à Garaison 1917, Toulouse, Le Pérégrinateur.

\section{Études critiques}

Audoin-Rouzeau, Stéphane / Becker, Annette (2000), 14-18 Retrouver la guerre, Paris, Gallimard.

Audoin-Rouzeau, Stéphane / Becker, Jean-Jacques (dir.) (2004), Encyclopédie de la Grande Guerre 1914-1918, Paris, Bayard.

Banoun, Bernard (1998), "Une opérette 'sérieuse' ? À propos du Baron tsigane (1885) de Johann Strauß fils », in Austriaca, $n^{\circ} 46$, 75-89. 
Baranello, Micaela (2014), The Operetta Empire: Popular Viennese Music Theater and Austrian Identity 1900-1930, Dissertation presented to the faculty of Princeton University.

Bilodeau, Louis (2019), Jacques Offenbach, mode d'emploi, L'Avant-Scène opéra, Paris, Éditions Premières Loges.

Cazals, Rémy (dir.), Dictionnaire et guide des témoins de la Grande Guerre, en ligne, http://www.crid1418.org/temoins.

Csáky, Moritz (1998²), Ideologie der Operette und Wiener Moderne: ein kulturhistorischer Essay, Wien, Böhlau.

Cubero, José (2017), Le camp de Garaison. Guerre et nationalités 1914-1919, Pau, CAIRN.

Filhol, Émmanuel (2004), Un camp de concentration français. Les tsiganes alsaciens-lorrains à Crest 1915-1919, Grenoble, Presses universitaires de Grenoble.

Klotz, Volker $\left(2004^{3}\right)$, Operette. Porträt und Handbuch einer unerhörten Kunst, Kassel, Basel u.a., Bärenreiter.

Köbner, Gertrud / Schaarschmidt, Helene (2016), Récits de captivité. Garaison 1914, textes édités par Hilda Inderwildi et Hélène Leclerc, Toulouse, Le Pérégrinateur.

Latzko, Andreas (1918), Frauen im Krieg. Geleitworte zur Internationalen Frauenkonferenz für Völkerverständigung in Bern, Zürich, Max Rascher Verlag A.-G.

Leclerc, Hélène (dir.) (2018), Le Sud-Ouest de la France et les Pyrénées dans la mémoire des pays de langue allemande, Toulouse, Le Pérégrinateur.
Leclerc, Hélène (2020), « Diaries of Women in Captivity. The Internment of German and Austrian Civilians during the First World War as Depicted in the Testimonies of Gertrud Köbner, Helene Schaarschmidt and Helene Fürnkranz », in Revue Belge de Philologie et d'Histoire, 98 (3), 69-88.

Thébaud, Françoise (1986/2013), La femme au temps de la guerre 14, Paris, Payot \& Rivages.

Vimont, Jean-Claude (1997), « La population du camp d'internement de Garaison (Hautes-Pyrénées), 1914-1919 », in Corvisier, André / Jacquart, Jean (ed.), Les malheurs de la guerre II. De la guerre réglée à la guerre totale, Paris, Éditions du CTHS, 93-108.

Vimont, Jean-Claude (2012), « Garaison, un camp de familles internées dans les Hautes-Pyrénées (1914-1919) », in Criminocorpus [http://journals.openedition.org/criminocorpus/1876, consulté le 13/01/2021].

Yon, Jean-Claude (2000), Jacques Offenbach, Paris, Gallimard.

\section{Sitographie}

http://creg.univ-tlse2.fr

https://blogs.univ-tlse2.fr/garaison

http://www.crid1418.org/temoins

https://ruhlebenlofthouse.com/ 